\title{
Long-term survival of patients with univentricular heart not treated surgically
}

\author{
Alfred Hager, MD, PhD, Harald Kaemmerer, MD, VD, PhD, Andreas Eicken, MD, PhD, Sohrab Fratz, MD, PhD, and \\ John Hess, MD, PhD, Munich, Germany
}

$\mathrm{U}$ niventricular heart (UVH) is a rare congenital heart malformation. Prognosis without surgical intervention is considered to be poor, and survival into late adulthood is unusual. ${ }^{1,2}$ Therefore if feasible, separation of systemic and pulmonary circulation by means of total cavopulmonary connection is the goal during childhood. However, long-term results after a Fontan-type operation are still associated with significant mortality and morbidity caused by atrial arrhythmia, venous congestion, protein-losing enteropathy, thrombembolism, and ventricular failure. ${ }^{3,4}$ For selected patients, ventricular septation is an option, but surgical mortality is high. ${ }^{5}$ Nevertheless, on long-term follow-up, cardiorespiratory responses to exercise seem to be more favorable than in those patients with a Fontan-type operation. ${ }^{6}$

However, in some patients the native hemodynamic situation warrants a long-term outlook that probably exceeds that after surgical interventions. To illustrate this, we report on a 62-year-old woman with UVH, L-transposition of the great arteries, and pulmonary outflow obstruction who did not undergo surgical intervention and on an additional 9 adults with UVHs from the literature who survived beyond the age of 50 years without an operation to get an impression about survival options and quality of life. Factors that might contribute to this extraordinary long-term survival are outlined.

\section{Patients and Methods}

A 62-year-old white woman with double-inlet left ventricle, ventriculoarterial discordance (L-transposition of the great arteries), and pulmonary and subpulmonary stenosis was admitted to our institution.

Shortly after birth, a congenital heart defect was suspected. Cardiac catheterization at 21, 28, and 39 years established the complete diagnosis. Growth and development during infancy and childhood were considered normal. Up to the age of 22 years, she participated in sports with only slightly reduced exercise tolerance.

\footnotetext{
From the Department of Pediatric Cardiology and Congenital Heart Disease, Deutsches Herzzentrum München, Technische Universität München, Munich, Germany.

Received for publication Oct 19, 2001; accepted for publication Jan 1, 2002.

Address for reprints: A. Hager, Department of Pediatric Cardiology and Congenital Heart Disease, Deutsches Herzzentrum München, Technische Universität München, Lazarettstr 36, D-80636, Munich, Germany (E-mail: hager@dhm.mhn.de).

J Thorac Cardiovasc Surg 2002;123:1214-7

Copyright (C) 2002 by The American Association for Thoracic Surgery $0022-5223 / 2002 \$ 35.00+0 \quad \mathbf{1 2 / 5 4 / 1 2 2 5 3 5}$

doi: $10.1067 / \mathrm{mtc} .2002 .122535$
}

At the age of 25 and 27 years, she gave birth to 2 healthy children without complications. Two episodes with signs of cardiac failure and increased dyspnea and cyanosis during exercise at the age of 27 and 44 years, respectively, were successfully treated with digoxin administered for a short time. This was the case until 3 years ago, when she experienced persistent atrial fibrillation and began permanent treatment with digoxin. Up to the present, she has lived at home, managing her household alone. Recently, she presented with further decreasing exercise tolerance and increasing cyanosis.

Physical examination showed moderate cyanosis and clubbing of the digits. Hydration status was balanced. The jugular venous pressure appeared normal. Arterial pulses were normal. At rest, blood pressure was $145 / 80 \mathrm{~mm} \mathrm{Hg}$, and heart rate was 65 beats/ min. The lungs were clear. A left-sternal-edge impulse was not observed. The apex of the ventricle was dislocated inferiorly and to the left. There was a systolic thrill in the second and third left-sided parasternal intercostal spaces. The liver and spleen were not enlarged, and there was no peripheral edema. Bilateral varicosis was seen at both legs. On auscultation, the first sound was normal, and the second heart sound was single and loud in the second left intercostal space. There was no ejection click. An apical third heart sound was also audible. A grade 4 to 5/6 systolic ejection murmer could be heard at the upper left sternal border.

Electrocardiography showed atrial fibrillation, with a ventricular rate of 60 to 80 beats/min, multiple polymorphic ventricular ectopic beats, intermittant bigemini, and nonsustained ventricular runs. The QRS axis was to the right (mean axis $+110^{\circ}$ ), and no $\mathrm{Q}$ waves were seen in the left precordial leads. There was a horizontal ST-segment depression in the inferior leads.

Chest radiography. A markedly enlarged heart (cardiothorax ratio of 0.68) with an enlarged left atrium and a small notch at the left cardiac border was present. The upper left cardiac border was convex. The pulmonary arteries were markedly enlarged (Figure 1).

Pathologic laboratory findings. Transcutanous oxygen saturation was $88 \%$. The hemoglobin level was $18.9 \mathrm{~g} / \mathrm{dL}$, hematocrit $58 \%$, mean corpuscular hemoglobin $32.6 \mathrm{pg}$, uric acid $7.3 \mathrm{mg} / \mathrm{dL}$, and serum gamma-glutamyl transferase $37 \mathrm{U} / \mathrm{L}$. Normal serum iron level was $183 \mu \mathrm{g} / \mathrm{dL}$, normal transferrin was $2.7 \mathrm{~g} / \mathrm{L}$, and normal ferritin was $116 \mathrm{ng} / \mathrm{mL}$.

Morphologic findings on the basis of transthoracic and transesophageal echocardiography, magnetic resonance imaging, and cardiac catheterization. Morphologic findings included situs solitus of the viscera and atria; normal systemic and pulmonary venous connection; patent foramen ovale; 2 atrioventricular valves (with a mild regurgitation of the left-sided atrioventricular valve) connected to a single, morphologically left ventricle; unrestrictive ventricular septal defect (outlet foramen) to a small right ventricular outlet chamber located anteriorly and to the left; ventriculoarterial discordance, with a left-sided aorta arising from the outlet 

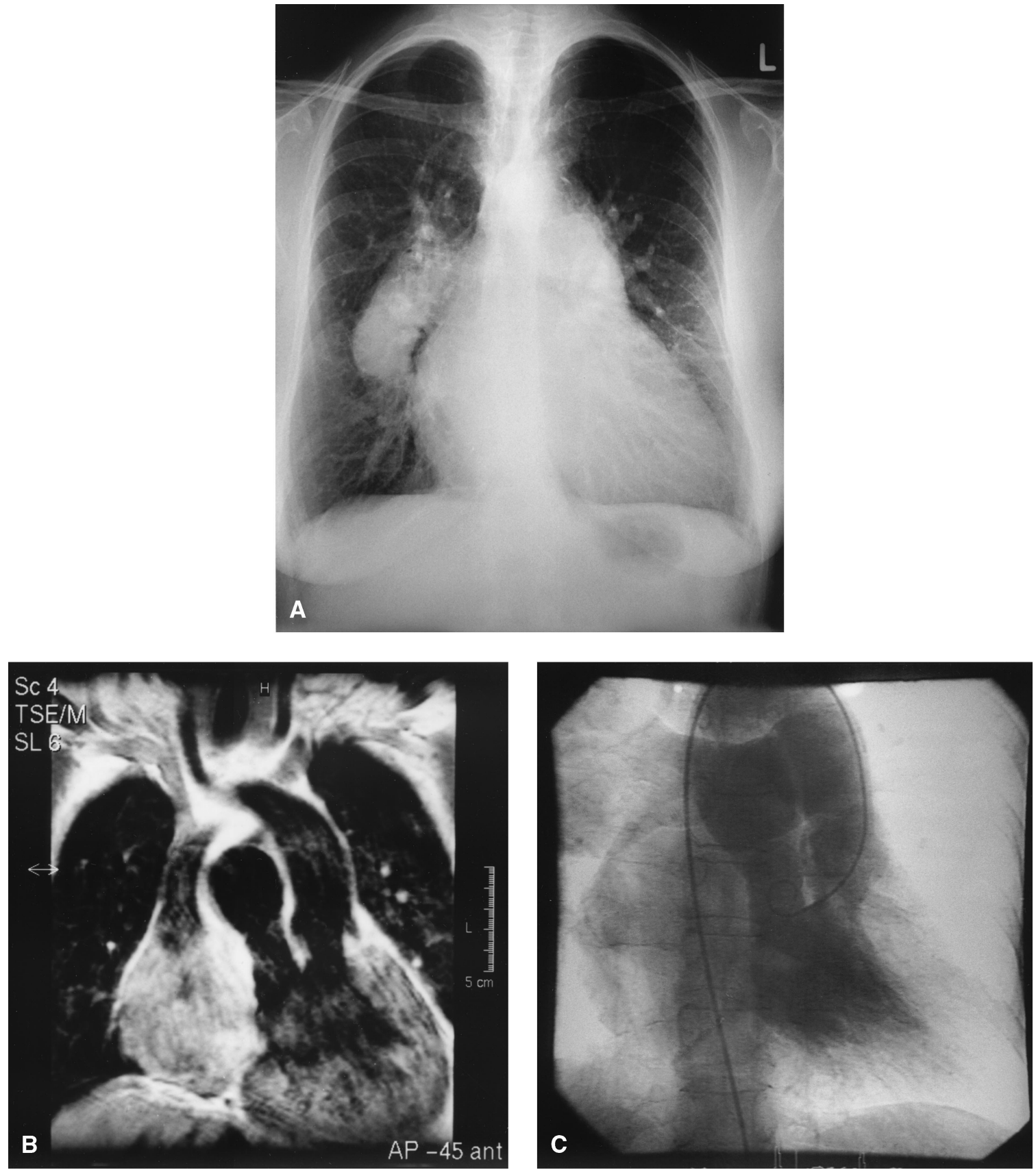

Figure 1. Chest radiograph (posteroanterior view), magnetic resonance image (frontal view), and ventriculogram (posteroanterior view) of a 62-year-old woman with double-inlet left ventricle, L-transposition of the great arteries, and subpulmonary and pulmonary stenosis.

chamber without any obstruction and a right-sided pulmonary artery connected to the morphologically left ventricle; and fibrous continuity of the bicuspid stenotic pulmonary valve to the rightsided atrioventricular valve, building a ridge-like fibromuscular subvalvular pulmonary stenosis.

Hemodynamic findings at cardiac catheterization. Cardiac index was $4 \mathrm{~L} \cdot \min ^{-1} \cdot \mathrm{m}^{-2}$, and the pulmonary blood flow (Qp)/ systemic blood flow ratio was 2.3:1. Mixing of arterial and venous blood in the single ventricle was complete. Mean pressure in the pulmonary artery was $38 \mathrm{~mm} \mathrm{Hg}$, pulmonary vascular resistance (Rp) was 3 Wood units $\cdot \mathrm{m}^{2}$, and systemic vascular resistance was 17 Wood units $\cdot \mathrm{m}^{2}$.

During symptom-limited exercise testing on a bicycle ergometer while in a sitting position (World Health Organization protocol: begin at $25 \mathrm{~W}$ with a stepwise increase of $25 \mathrm{~W}$ every 2 minutes), the patient reached $75 \mathrm{~W}$, with only a minor decrease of the 
TABLE 1. Reports on patients with UVH not treated surgically surviving beyond the age of 50 years

\begin{tabular}{llll}
\hline First author & Age/sex & \multicolumn{1}{c}{ Type } & Clinical condition \\
\hline Goldberg, 19837 & $62 / \mathrm{M}$ & DILV, L-TGA, PS & Died \\
Oliver, 19908 $^{\text {Habeck, 19919 }}$ & $61 / \mathrm{F}$ & DILV, L-MGA, PHT & Mild symptoms \\
Koito, 199410 & $59 / \mathrm{M}$ & DILV, D-TGA, PHT & Died \\
Warner, 199611 & $57 / \mathrm{M}$ & DILV, L-TGA, PHT & Alive \\
Ammash, 199613 & $61 / \mathrm{F}$ & DILV, L-TGA, PS, endocarditis & Surgical treatment \\
Ammash, 199613 & $60 / \mathrm{M}$ & DILV, ?-TGA, PS & Died \\
Ammash, 199613 & $60 / \mathrm{M}$ & DILV, ?-TGA, PS & Mild symptoms \\
Vitarelli, 199612/Gabbarini, 199914 & $66 / \mathrm{M}$ & DILV, ?-TGA, PS & Mild symptoms \\
Present report & $61 / \mathrm{M}$ & DILV, D-MGA, PHT & Fairly good \\
\hline
\end{tabular}

DILV, Double-inlet left ventricle; $L-T G A / D-T G A$, transposition of the great arteries with a left/right position of the aorta; $L-M G A / D-M G A$, ventriculoarterial concordance with a left/right position of the aorta; $P H T$, pulmonary hypertension; $P S$, pulmonary stenosis.

oxygen saturation from $89 \%$ to $83 \%$ and an increase of the ventricular heart rate from 64 to 125 beats/min.

We recommended restoration of sinus rhythm by means of cardioversion after pretreatment with amiodarone and coumadin. The patient was discharged with these recommendations, but she did not comply because of fear of adverse side effects. Currently, 6 months later, she is quite well.

Additionally, a literature search of the last 10 years was performed with the topics of "univentricular heart," "single ventricle," and "double-inlet ventricle." In reports on long-term survival of patients with UVH not undergoing surgical treatment, the references were also checked. We found another 9 patients with UVH who were older than 50 years. ${ }^{7-14}$ Their anatomic situation is listed in Table 1.

\section{Discussion}

UVH is a rare congenital cardiac anomaly occurring in $1 \%$ to $2 \%$ of all congenital heart defects in newborns. ${ }^{15}$ Male patients predominate. Different nomenclatures exist (eg, Anderson and van Praagh). Depending on the ventricular morphology, the single ventricle can be subdivided as left ventricular type (60\%-66\%), right ventricular type $(10 \%-24 \%)$, or indeterminate type $(<10 \%) .{ }^{16}$ The great vessel connections are variable, being concordant in $15 \%$ and discordant (D- or L-transposition) in $85 \% .{ }^{17}$ Frequently, a subvalvular or valvular outflow tract obstruction to either great artery is present. Other associated anomalies are hypoplastic ascending aorta, coarctation, patent arterial duct, anomalies of the atrioventricular valves, and heterotaxy syndromes.

The most common form of UVH consists of a single left ventricle with a subaortic right ventricular outflow chamber. Only about $30 \%$ of patients with UVH reach the age of 16 years. ${ }^{2}$ More recently, survival was estimated to be only $30 \%$ for the the first year of life. ${ }^{1}$ Survival into late adulthood is exceptional, and patients reaching even their sixties without a cardiovascular operation are rare.

The clinical presentation and long-term outlook ${ }^{16}$ depend on the presence or absence of an obstruction to the pulmonary blood flow and the pulmonary vascular resistance, morphology, and function of the ventricle; obstruction to aortic flow; and morphology and function of the atrioventricular valves.

Pulmonary outflow obstruction and Rp determine Qp and leftto-right shunting.
In severe pulmonary stenosis Qp is reduced, leading to a normal-sized or small heart and severe systemic arterial desaturation. Most of these patients die during infancy or require surgical treatment in early infancy.

In moderate pulmonary stenosis Qp is nearly normal, leading to no or mild heart failure, mild systemic arterial desaturation, and no pulmonary arteriolar disease. Long-term survival is possible with an adequate oxygenation and balanced volume load of the ventricle.

In patients without pulmonary stenosis, Qp depends on Rp. If $\mathrm{Rp}$ is low, patients experience severe cardiac failure, leading to death in infancy. If the patient had pulmonary arteriolar disease (Eisenmenger reaction) and $\mathrm{Rp}$ is high, survival into adulthood is possible.

Morphology and function of the ventricle constitute another important issue. There are no reports of long-term survival with a UVH of the right or indeterminate type. Although never studied in detail, this is obviously caused by the intrinsic capacity of the left ventricle to deal with the systemic resistance. However, optimal diastolic function is also necessary to deal with volume changes during exercise. Congestive cardiac failure occurs in cases of volume overload that might result from excessive pulmonary blood flow, as already mentioned, and from regurgitant atrioventricular and semilunar valves.

Obstruction to aortic flow occurs at the level of the aortic valve or the ventricular septal defect in case of a subaortic right ventricular outflow chamber. With age, the latter might decrease in size and, if so, might result in a critical subaortic obstruction. This additional ventricular afterload promotes ventricular hypertrophy that further tightens the ventricular septal defect. This vicious circle leads to low systemic output, hyperperfusion of the lungs, and, finally, cardiac death.

Survival is positively influenced by competent and separated atrioventricular valves. In atresia of one atrioventricular valve, venous return depends on the size of a foramen ovale or of an atrial septal defect, which might lead to congestion if the intra-atrial communication is restrictive. An incompetent common atrioventricular valve poses a volume overload to the ventricle. However, as already mentioned, separated atrioventricular valves with moderate regurgitation are also a volume hazard to ventricular function.

The most promising survival is seen in patients with a single ventricle of left ventricular morphology, transposition of the great 
arteries without systemic outflow obstruction, an adequately functioning atrioventricular valve, and a moderate pulmonary outflow obstruction.

The presented data demonstrate that patients with UVH and well-balanced pulmonary perfusion might survive into late adulthood with good quality of life and good functional capacity and without major symptoms or depression of cardiac function.

Older adults presenting with UVH and no prior surgical intervention have already proven to have an unexpectedly good longterm prognosis. In our opinion these patients should be followed and treated conservatively. Major cardiac surgery, such as cavopulmonary anastomosis, which leads to gross hemodynamic adjustments and which has a considerable morbidity and mortality in this age group, should be avoided. However, one should be careful to extrapolate these recommendations to children or infants because we cannot foresee the hemodynamic and clinical condition in late adulthood.

\section{References}

1. Samanek M. Children with congenital heart disease: probability of natural survival. Pediatr Cardiol. 1992;13:152-8.

2. Moodie DS, Ritter DG, Tajik AJ, O'Fallon WM. Long-term follow-up in the unoperated univentricular heart. Am J Cardiol. 1984;53:1124-8.

3. Fontan F, Kirklin JW, Fernandez G, et al. Outcome after a "perfect" Fontan operation. Circulation. 1990;81:1520-36.

4. Gatzoulis MA, Munk MD, Williams WG, Webb GD. Definitive palliation with cavopulmonary or aortopulmonary shunts for adults with single ventricle physiology. Heart. 2000;83:51-7.
5. Nagashima M, Imai Y, Takanashi Y, et al. Ventricular hypertrophy as a risk factor in ventricular septation for double-inlet left ventricle. Ann Thorac Surg. 1997;64:730-4.

6. Ohuchi H, Arakaki Y, Yagihara T, Kamiya T. Cardiorespiratory responses to exercise after repair of the univentricular heart. Int J Cardiol. 1997;58:17-30.

7. Goldberg HL, Sniderman K, Devereux RB, Levin A. Prolonged survival (62 years) with single ventricle. Am J Cardiol. 1983;52:214-5.

8. Oliver JM, Fdez de Soria R, Dominguez FJ, Ramos F, Calvo L, Ros J. Spontaneous long-term survival in single ventricle with pulmonary hypertension. Am Heart J. 1990;119:201-2.

9. Habeck JO, Reinhardt G, Findeisen V. A case of double inlet left ventricle in a 59-year-old man. Int J Cardiol. 1991;30:119-20.

10. Koito H, Ohkubo N, Suzuki J, Iwasaka T, Inada M. Prolonged survival in a patient with a single ventricle without pulmonary stenosis. Chest. 1994;106:971-2.

11. Warner KG, Payne DD, Fulton DR, et al. Excision of a pulmonary valve abscess in a 61-year-old woman with single ventricle. Ann Thorac Surg. 1996;61:213-5.

12. Vitarelli A, Gabbarini F. Holmes heart in the adult: transesophageal echocardiographic findings and long-term natural survival. Int J Cardiol. 1996;56:301-5.

13. Ammash NM, Warnes CA. Survival into adulthood of patients with unoperated single ventricle. Am J Cardiol. 1996;77:542-4.

14. Gabbarini F. A 61-year-old man with Holmes' heart. Lancet. 1999; 353:646.

15. Samanek M, Voriskova M. Congenital heart disease among 815,569 children born between 1980 and 1990 and their 15-year survival: a prospective Bohemia survival study. Pediatr Cardiol. 1999;20:411-7.

16. Perloff JK. The clinical recognition of congenital heart disease. 4th ed. Philadelphia: W.B. Saunders; 1994.

17. Van Praagh R, Ongley P, Swan H. Anatomic types of single or common ventricle in man: morphologic and geometric aspects of 60 necropsied cases. Am J Cardiol. 1964;13:367-86. 